\title{
ZONAS DIALECTALES DEL QUECHUA EN EL SUR DE ANCASH*
}

\author{
Gustavo Solís Fonseca
}

\section{RESUMEN}

Isoglosas fonológicas y morfológicas permiten reconocer en el quechua hablado en el sur de Ancash, en el ámbito que correspondía a la antigua provincia de Bolognesi, las siguientes formaciones dialectales más inclusivas: Quechua tipo Alto Pativilca, Quechua tipo Callejón de Huaylas, Quechua tipo Yarú y Quechua que conserva */č/ y */ĉ/. Estas formaciones dialectales han sido identificadas a partir del examen de las isoglosas morfológicas referidas al tratamiento de las marcas de segunda persona y del pluralizador verbal; como también de las isoglosas fonológicas siguientes: monoptongización de las secuencias /ay/, /iy/ y /aw/, desafricación de $* / c /$, adelantamiento de $* / \hat{c} /$ y despalatalización de $\mathrm{hl} / \mathrm{y} / \tilde{\mathrm{n}} / \mathrm{l}$.

Los datos permiten identificar al río Pativilca como una frontera dialectal muy antigua entre una variedad quechua tipo Yaru y otra tipo Huaylas. Asimismo, los indicios lingüisticos señalan que la presencia de elementos quechuas tipo Cajatambo en el sur de Ancash, al otro lado del río, es relativamente reciente; pero también se observa una vuelta del habla quechua de la zona a su matriz primigenia tipo Huaylas.

\section{ABSTRACT}

Phonologic and morphologic isoglosses let us recognize in the spoken Quechua from the south of Ancash, in the territory which belonged to the ancient Bolognesi province, the following more exclusive dialectic formations: Alto Pativilca Quechua, Callejón de Huaylas Quechua, Yaru Quechua, and a Quechua that preserves $* / c /$ and $* / \hat{c} /$. These dialect variants have been identified according to the analysis of the morphologic isoglosses referred to the treatment of the second person marks and the verbal pluralizer; so the following phonologic isoglosses: vocal cluster of the sequences /ay/, /iy/ and /aw/, "desafricación" of $* / c /$, fronting of $* / \hat{c} /$ and dispalatalization of $\Lambda \mathrm{L}$ and $/ \tilde{n} /$.

The data allowed us identify Pativilca River as a very old dialect border between a Yaru Quechua variety, and another Huaylas kind. In the same manner the linguistic clues indicates the presence of Quechua elements Cajatambo kind in the south of Ancash, at the other side of the river, is relatively recently; but also we can see a return of Quechua language in the zone of its primigenial matrix Huaylas kind.

\footnotetext{
* Este artículo es una versión resumida de parte de mi tesis Zonificación (dia)lectal del quechua de la provincia de Bolognesi (Ancash) de 1976. El comportamiento de las isoglosas y la zonificación dialectal resultante corresponden a la época en que se hicieron los estudios.
}

Arqueología y Sociedad $\mathrm{N}^{\circ}$ 14, 2002: 151-164 
$\mathrm{E}$ n este trabajo presentamos una zonificación' del quechua hablado en el sur del departamento de Ancash. Lo que aquí identificamos como sur de Ancash coincide con el espacio geográfico que tenía la provincia de Bolognesi antes de su división para dar lugar a la provincia de Ocros. La zonificación resultante nos permite ver que las variedades más significativas -incluso reconocidas intuitivamente por los propios hablantes- coinciden con subdivisiones menores establecidas por reconocidos especialistas dentro del llamado Quechua I o Quechua B².

\section{IMPORTANCIA LINGÜÍSTICA DE LA ZONA}

La zona del sur de Ancash es, respecto del quechua, de gran interés dialectológico. Entre las principales razones de este interés mencionaremos las siguientes:

a. Se presume que este territorio habría sido ocupado por el quechua en forma continua desde el momento de la difusión del protoidioma (desde su cuna ubicada en la zona costeña contigua, Valle del río Pativilca, o norte chico). El sitio arqueológico de Caral (Supe) habría sido el centro político desde donde el protoquechua se extendió.

b. Constituye un área poco estudiada por los especialistas, en comparación con otros lugares.

c. Es una región lingüística donde se presentan particularidades morfológicas importantes y confluyendo diversos procesos de cambios fonológicos que singularizan a la lengua quechua hablada en la zona.
Con relación al punto (c) cabe señalar, por ejemplo, que dicha región se encuentra en el límite suroeste del departamento. Hasta esta zona ha llegado la monoptongación iniciada en el Callejón de Huaylas. En esta zona, posiblemente, se encontrarían las manifestaciones del cambio en proceso. Por otro lado, observamos que por los alrededores del poblado de Mangas es productivo el alargamiento vocálico, de manera sincrónica, o lo que podría ser un nuevo ciclo de este proceso particularmente importante en el quechua central. Igualmente, el tratamiento del protofonema /q/, de muy variada manifestación en el territorio de la provincia, y muy especialmente en el distrito de San Francisco de Mangas, es otro fenómeno de cambio bastante interesante. El tratamiento de /q/ en este distrito se relaciona con el que recibe en la Sierra Central, especialmente en Tarma. Procesos detenidos, como la despalatalización de / $\tilde{n} / \mathrm{y}$ de $/ 1 \mathrm{l} /$, que no ha llegado a Cajamarquilla, en la margen izquierda del río Pativilca, donde sí se ha dado el cambio de las africadas y otros cambios particulares, son otras tantas características que hacen singular a la zona en cuestión.

En el aspecto morfológico, el quechua hablado en este territorio presenta una importante isoglosa relacionada con el tratamiento del morfema de segunda persona actora y poseedora que es la forma única /-yki/ en el distrito de San Francisco de Mangas y sus caseríos; mientras que en el resto de los lugares son formas diferentes para la persona actora y para la persona poseedora: /-nki/ e /-yki/, según contextos para persona actora; e /-yki/ para persona poseedora. Esta diferencia en el tratamiento de la segunda persona, que va aparejada a otras diferencias 
de orden morfológico, permite agrupar al quechua de la zona en dos lectos mayores:

a.- Aquel que usa sólo /-yki/ para actor y poseedor.

b.- Aquel que emplea /-nki/ e /-yki/ para actor, e /-yki/ para persona poseedora.

\section{UBICACIÓN DE LA ZONA}

El territorio ocupado queda al suroeste del departamento de Ancash. Coincide con el territorio que tenía la provincia de Bolognesi antes de su desmembración. Los límites políticos del espacio en cuestión son: al norte y noreste con las provincias de Casma, Recuay y Huari; al este con la provincia huanuqueña de Dos de Mayo; y al sur las provincias limeñas de Cajatambo y Chancay. Ninguna parte de este territorio conforma el Callejón de Huaylas ni el Callejón de Conchucos; estos dos valles tienen gran importancia geopolítica y sociolingüística en el departamento. El valle del río Santa tiene su inicio en la laguna de Conococha que se localiza exactamente en la línea fronteriza con Recuay, al norte de Bolognesi, en la Collana de Lampas. Toda esta zona involucrada queda al oeste de la Cordillera Blanca; la Cordillera Negra la atraviesa de sur a norte. Una gran parte de este territorio está en las vertientes occidentales de la Cordillera $\mathrm{Negra}^{3}$.

La vía más importante del departamento anteriormente el principal camino incaico y colonial - se inicia en Pativilca (Lima), recorre el valle del río Fortaleza para tomar luego la vera del río Santa. Una divisoria que sale de Conococha lleva a Chiquián, capitál de la provincia de Bolognesi.

\section{ISOGLOSAS}

La zonificación que presentamos se sustenta en el tratamiento de las isoglosas morfológicas y fonológicas que figuran enseguida:

\section{Morfológicas}

a. Morfema de referencia a la segunda persona

b. Pluralizador verbal

\section{Fonológicas}

a. Tratamiento de /q/

b. Monoptongación de las secuencias /ay/, /uy/, /aw/

c. Alargamiento vocálico

d. Despalatalización de /l1/, /ñ /

e. Desafricación de /č/, /čl

Muy brevemente vamos a describir en los párrafos que siguen el comportamiento de las isoglosas señaladas en el quechua del sur de Ancash.

\section{ISOGLOSAS MORFOLÓGICAS}

\section{Morfema de referencia a la segun- da persona}

Las formas más difundidas de la marca de referencia a la segunda persona en el quechua general son /-nki/ e /-yki/. Existe una cierta especialización de estos sufijos, pues el primero se usa en la frase verbal, en tanto que el segundo se emplea como marca de persona en la frase nominal, en la que se hace referencia semántica a la persona poseedora. En ciertas variedades quechuas /-yki/ es la única marca para ambas funciones; en tanto que en otras, en la frase verbal se emplea en distribución complementaria tanto /-nki/ e /-yki/. Esta forma parece que se concretiza algunas veces como /-ki/ (Cajamarca: yawar-ki "tu sangre"); o como /-y/ (Bolognesi: munayman "quisieras"). Procesos fonológicos que afectan principalmente a $/ \mathrm{k} /$ hacen que la marca de segunda persona presente distintas configuraciones fonéticas. Secuencias tales como [-nkyi] [-ykyi]; [-ntyi] [-ytyi]; [-nchi] [-ychi] 


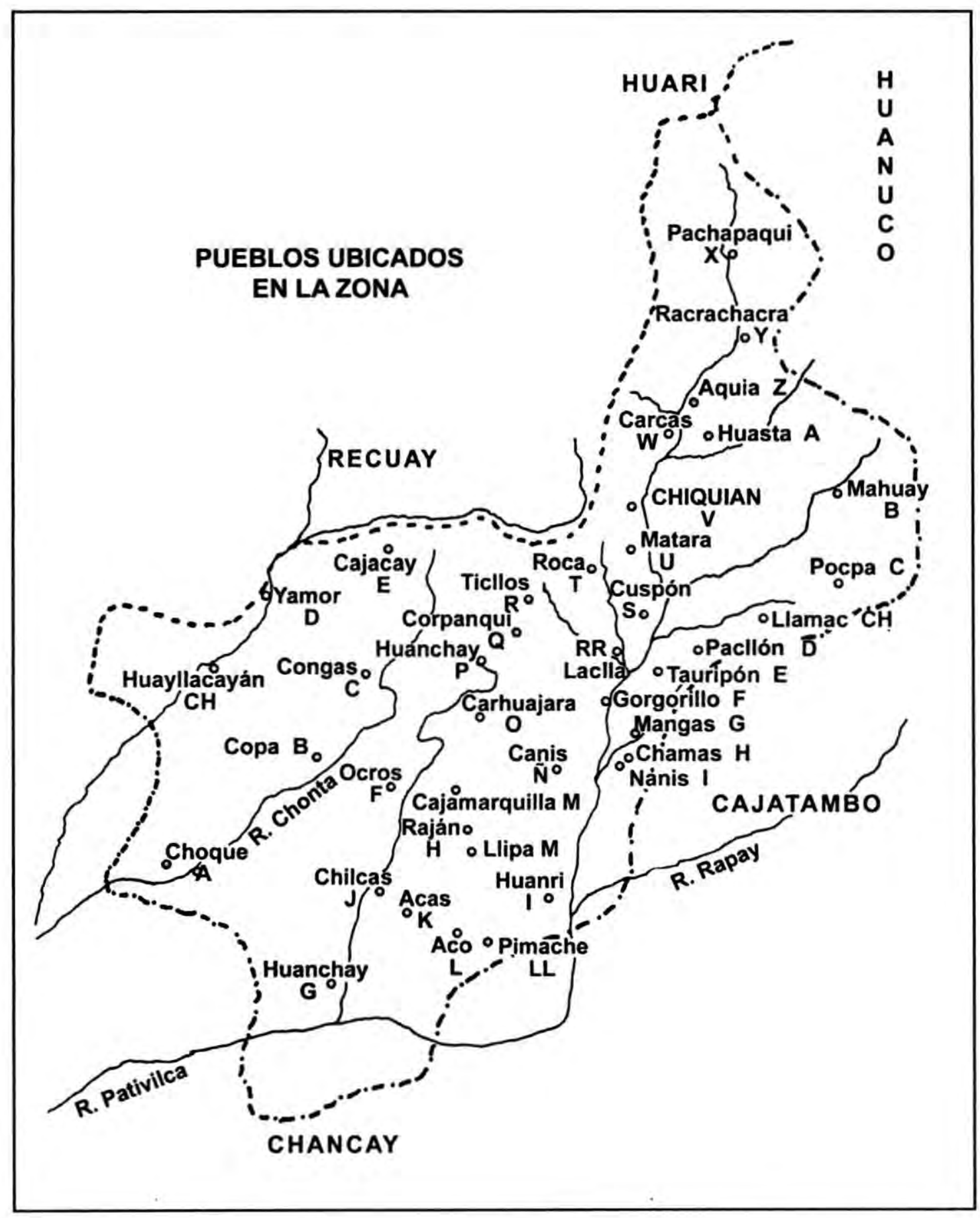

Figura 1. Pueblos ubicados en la zona de estudio. 
son manifestaciones fonéticas de las formas -nki e -yki, respectivamente, y muestran la amplia gama de variación fonética que ocurre con - $\mathrm{k}$ en dichas marcas de segunda persona actora y poseedora. Se puede constatar también la forma /-y/ como señal de segunda persona en algunas hablas de Bolognesi (Ej. munayman "quisieras").

\section{Pluralizador verbal}

Existen en el quechua varios sufijos en la palabra verbal cuya función semántica es expresar la idea de pluralidad referida al sujeto o al objeto de la acción verbal. En el caso del Quechua I tenemos el siguiente inventario de formas:

\section{I-ya:/ \\ /-rka:/ \\ /-pa:ku/, /-ba:ku/. \\ /-:ri/ \\ /-raqari/}

En el quechua de Ancash figuran comúnmente /-ya:/, /-rka:/ y /-rqari/. Las demás formas están presentes en el quechua de Junín. La forma /-ya:/ es de uso más generalizado en Bolognesi y en general en todo Ancash. La forma /-rka:/ solamente se comprueba en Bolognesi y tiene una distribución variada. Mientras que la forma /-rqari/ se menciona para el Callejón de Huaylas. En la parte sur de Bolognesi (límite con Cajatambo), /-rka:/ es el único sufijo pluralizador; entre tanto que en el resto de la provincia hay alternancia de /-rka:/ /-ya/.

Respecto a esta alternancia es posible señalar que /-rka:/ está perdiendo terreno a favor de /-ya:/, lo que a nuestro parecer evidenciaría el ingreso reciente de esta última forma, desplazando a /-rka:/, la misma que se mantiene sólo en los pueblos pertenecientes al distrito de San Francisco de Mangas, fronterizos con la provincia limeña de Cajatambo.

\section{ISOGLOSAS FONOLÓGICAS}

\section{Tratamiento de /q/}

Este segmento presenta el más amplio espectro de variación fonética sincrónica. Por ejemplo, Torero (1964) presenta hasta siete realizaciones distintas de esta consonante. Cerrón-Palomino (1973) anota que los cambios ocurridos al protofonema /* $\mathrm{q} /$ son los que caracterizan al wanka respecto a las otras variedades quechuas. En la mayor parte del territorio de Ancash hay estabilidad en la realización "originaria" postvelar oclusiva sorda de /q/, con excepción a la zona sur del departamento, donde parece darse una confluencia de procesos de cambios que se dan en el quechua tipo Wanka y en el tipo de quechua hablado en el Callejón de Huaylas.

\section{Monoptongación}

La monoptongación es el cambio fonológico que caracteriza la fisonomía fonética del quechua de Ancash, especialmente de las variedades habladas en el Callejón de Huaylas (Pantoja, S. et al 1974). Este cambio consiste en la coalescencia de las secuencias homosilábicas (diptongos) de vocal más semiconsonante en monoptongos. Las secuencias que sufren este cambio son:

\section{lay/ /uy/ liy/ law/}

Un quinto diptongo /iw/, no muy frecuente en la lengua, no está sujeto a monoptongación. La lengua no permite la vigencia del diptongo /uw/ por regla de estructura morfémica.

Hay una serie de etapas en la realización fonética de cada uno de los diptongos desde el inicio del proceso hasta su realización completa como monoptongo. Por otro lado, cabe señalar que el proceso se inicia en límite de sufijo, entre: 
... a+y..., a+w...

... u+y...

... i $\mathrm{i}+\mathrm{y} .$.

La generalización de la innovación consiste en borrar el signo de linde de morfema para luego atacar toda secuencia /ay/, /uy/, /iy/, /aw/ que presenta la lengua.

\section{Alargamiento vocálico}

La aparición de cantidad vocálica es una de las innovaciones fonológicas más remotas atestiguable en el idioma quechua. La idea es que la cantidad vocálica se origina de un segmento o de una secuencia de segmentos, como por ejemplo el segmento /y/ de la protolengua, que sola o adyacente a vocal (precedente o siguiente) se funde en una única vocal que es fonéticamente Iarga, como se puede observar en muchos términos cognados de varios dialectos del quechua (Adelaar 1970).

Son varios los segmentos que producen sincrónicamente cantidad vocálica. Entre éstos tenemos:

$/ \mathrm{y} /$

/q/

$/ \mathrm{n} /$

$/ \mathrm{w} /$

De los cuatro segmentos señalados, /y/ es altamente productivo, le sigue en productividad la consonante /q/.

La presencia de cantidad vocálica en el quechua así como su análisis se explica muy bien en el marco de la hipótesis de Brent de Chene (1979).

En esta perspectiva, cabe anotar que la elisión del protofonema */y/ es la fuente más remota comprobable de surgimiento de cantidad vocálica en quechua. Por otro lado, debe señalarse que no toda presencia de alargamiento vocálico es secuela de la elisión de consonante. Los datos hacen ver que en esta lengua se dio un proceso de reanálisis funcional, el cual hizo que se interpretara como vocal larga algunas vocales de la lengua que, previamente, eran cortas.

\section{Despalatalización de /11/ y de /ñ/}

Aun cuando hay contemporaneidad parcial en la despalatalización de los fonemas / $\tilde{n} / \mathrm{y}$ $\mathrm{hl} \mathrm{l}$, hay varios indicios que revelan la mayor antigüedad de la despalatalización de /ñ/. Por ejemplo, no existe alternancia en la despalatalización de /ñ/; en cambio sí existe en la realización de /11/, pues es comprobable $\{11,1 y, 1\}$. En ambos casos la despalatalización se inicia en límite de sufijo, para luego extenderse a raíces.

\section{Desafricación de /čl y de /ć/}

Las africadas de la protolengua, $/ * c ̌ /$ y $/ * \hat{c} /$, han tenido diferente destino en el quechua. En la mayor parte de las variedades $/ *^{*} \mathrm{c} /$ $\rightarrow / c ̌ /$, mientras que / $*$ č/ aparece, dependiendo de las variedades, sea como/ts/ (Huaraz) o como /s/ (Bolognesi y Ocros).

\section{ZONIFICACIÓN DIALECTAL}

El tratamiento de las isoglosas a las que hemos hecho referencia en el presente trabajo, así como la intuición de los hablantes acerca de las diferencias dialectales (Carrillo Ramírez 1953), nos permite hacer la siguiente zonificación dialectal del quechua del sur de Ancash, recogiendo las más importantes diferencias dialectales:

\section{ZONA DIALECTAL I}

El distrito bolognesino de San Francisco de Mangas, con su capital Mangas, y sus anexos: Chamas, Nanis, Gorgorillo y Tauripón y el distrito de Pacllón (con ex- 
cepción de sus anexos de Llamac y Pocpa) constituyen una zona dialectal bien definida. Esta zona se caracteriza por el uso del pluralizador + /-rka:/o de /-ya:/; así como del morfema de segunda persona +/-yki/. De igual manera, esta zona realiza las mayores innovaciones fonológicas del fonema /q/ y mantiene productivo el alargamiento vocálico. Respecto al proceso de la monoptongación, sólo lo cumple en los sufijos /-čaw/ y /-naw/. Esta área despalataliza /ñ/ y $/ 1 \mathrm{l} / \mathrm{en}$ todos los ambientes y ha cambiado $/ * \hat{c} /-->/ \check{c} / \mathrm{y} / * \mathrm{c} / \mid->/ \mathrm{s} /$.

En la clasificación de Alfredo Torero (1974), el habla de esta zona aparecería como perteneciendo a la variedad Yaru, a la que también incumbe la parte adyacente a Mangas de la provincia de Cajatambo, Lima.

Las hablas quechuas de Pacllón y el distrito de San Francisco de Mangas están estrechamente emparentadas con las de la ciudad de Cajatambo. El cambio de palatalización de $\mathrm{k} /$ que ocurre en esta zona, y que cumple con los siguientes pasos:

a. $/ \mathrm{k} / \rightarrow[\mathrm{ky}] /\left\{\begin{array}{c}\mathrm{i}(\mathrm{n}) \\ \mathrm{y}\end{array}\right\}-$

b. $/ \mathrm{k} / \rightarrow$ ty] $/ \mathrm{y}-$,

está relacionado con el cambio más avanzado:

$/ \mathrm{k} / \mathrm{- \rightarrow}$,

que ha ocurrido en el quechua de la ciudad de Cajatambo y poblados cercanos a Bolognesi, tales como Copa, Pocquián, etc.

En el pueblo de Mangas se observa la palatalización de $/ \mathrm{k} /$ en su forma más avanzada. Ha realizado los siguientes cambios:
a. $/ \mathrm{k} /-\rightarrow[\mathrm{ky}] / \mathrm{y}-$

$$
\begin{aligned}
& ->[k y] / i- \\
& ->[k y] / i(n)-
\end{aligned}
$$

b. $/ \mathrm{k} / \rightarrow[\mathrm{ty}] / \mathrm{y}-$

Solamente en el habla de los más jóvenes tenemos:

$/ \mathrm{k} / \mathrm{- \rightarrow}$ [ty] / i(n)__,

lo que constituye un mayor grado en el avance de la palatalización, es en este ambiente que en el habla de los adultos se realiza [ky].

En los pueblos de Nanis, Chamas, Gorgorillo y Tauripón (anexos del distrito de San Francisco de Mangas) y en Pacllón el proceso de cambios de $/ \mathrm{k} /$ cumple la siguiente regla:

$/ k / \rightarrow[k y] /\left\{\begin{array}{c}i(n) \\ y\end{array}\right\}-$

En el distrito de Mangas y el pueblo de Pacllón utilizan /-kama/ como morfema limitativo; la misma forma es usada en Cajatambo. En la variedad quechua de Huaraz se usa la forma /-yaq/.

En Mangas, Chamas y Nanis al igual que en el poblado vecino de Copa, perteneciente a la provincia de Cajatambo, se está iniciando una innovación fonológica que consiste en el cambio:

$/ \mathrm{n} /-\rightarrow[\mathrm{w}]-+\mathrm{m}$

$/ \mathrm{m} /-\rightarrow[\mathrm{w}]-+\mathrm{m}$

/kan+mi/ --> [kawmy] "hay"

/qan + man+mi/ - - > [qawmawmy] "hacia ti"

Otra característica lingüística de esta zona, que llama la atención a los hablantes de otras variedades del quechua de Bolognesi, es la realización del morfema/-pish/ que en otras variedades es $/$-pis $/{ }^{4}$. Ej. Paypish mikuykan "Él / ella también está comiendo". 


\section{ZONA DIALECTAL II}

Otra amplia zona dialectal está conformada por los distritos de Bolognesi cuyas capitales son los poblados de Aquia, Huasta, Chiquián, Ticllos, Corpanqui, Llaclla, Canis, Cajamarquilla y Llipa. La variedad de esta zona se caracteriza principalmente por: pluralizador + /-ya:/, + /-rka:/, este último morfema con una distribución restringida, pues ocurre solamente precediendo al morfema durativo /-yka:/.

El uso de la segunda persona en esta zona es del siguiente modo:

a.- /-yki/ en la frase nominal, y en la frase verbal sólo después de los sufijos /-rqa/, /-rqu/, I-na/, /shqa, -sha/. El quechua de algunos lugares de esta zona, después del sufijo /-rqu/ usa /-nki/.

\section{b.- /-nki/ en la frase verbal.}

En cuanto a las isoglosas fonológicas tenemos: fricación de /q/ en final de sílaba; en tanto que sonorización inicial, siendo oclusiva después de consonante nasal y en inicio de palabra y fricativa en posición intervocálica. La monoptongación ocurre únicamente con /aw/ y sólo en los sufijos /-čaw/ y /-naw/. La presencia de nuevas vocales largas se produce por el cambio de ciertas secuencias /ay/. Toda la zona ha hecho la

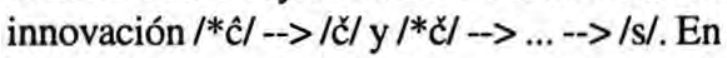
la mayor parte de los lugares de esta zona se despalatalizan $/ * \tilde{n} /$ y $/ * 1 \mathrm{l} /$, con excepción de Cajamarquilla, Canis, Llipa y Carhuajara donde se tiene $/ * \tilde{n} /-\rightarrow / n /$ sólo en sufijos y se conserva /11/ en todos los ambientes. En algunos lugares hay realización alternante de $/ * 11 /$--> [1l, ly, 1] pero solamente en raíces.

Según la clasificación de Torero (1974), esta zona formaría parte de la variedad quechua
Alto Pativilca- Alto Marañón - Alto Huallaga, (dialecto Alto Pativilca).

\section{ZONA DIALECTAL III}

Otra zona dialectal está formada por el territorio de los pueblos de Cajacay, Raquia, Yamor, Huayllacayán, Raján y Huanri (Bolognesi) y Congas, Copa, Choque, Huanchay (Ocros ). Esta zona maneja el pluralizador /-ya:/; algunas hablas como las de Cajacay, Yamor y seguramente las de otros lugares aceptan el pluralizador /-rka:/; en cambio, la de Huayllacayán lo rechaza tajantemente. Como morfema (sufijo) de referencia a la segunda persona usa /-yki/ y /-nki/ con la distribución respectiva ya vista anteriormente.

El fonema /q/ no sufre innovaciones, aunque en el habla informal y en final de sílaba hay la tendencia a pronunciarlo fricativo, por ejemplo en Congas, Copa, Huayllacayán. El alargamiento vocálico es estacionario en esta zona. No se presencia la despalatalización de $/ 1 \mathrm{l} /$. En lo que se refiere a / $\tilde{n} /$ parece haber diferente tratamiento; se despalataliza en los sufijos y, en Cajacay por ejemplo, los adultos no despalatalizan en raíces /ñuqa/ "yo"; en cambio, los más jóvenes sí lo hacen aunque con elevado número de excepciones. La consonante retrofleja africada

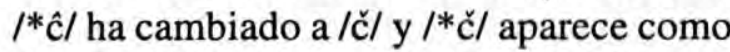
/ts/. En final de sílaba, y también al inicio en ciertas palabras, hay alternancia $/ \mathrm{ts} / \sim / \mathrm{s} /$. Ejemplos:

$$
\begin{aligned}
& \text { /lutsqa/ /lusqa/ "resbaloso" } \\
& \text { /tsay/ /say/ "eso" }
\end{aligned}
$$

Las hablas de esta zona ostentan las diferentes etapas del proceso de monoptongación. Por ejemplo, el habla de Huayllacayán no monoptonguiza, salvo los sufijos /-čaw/ y /-naw/y las palabras /čawpi/ "centro" y /nawpa/ "adelante"; la secuencia /ay/ 


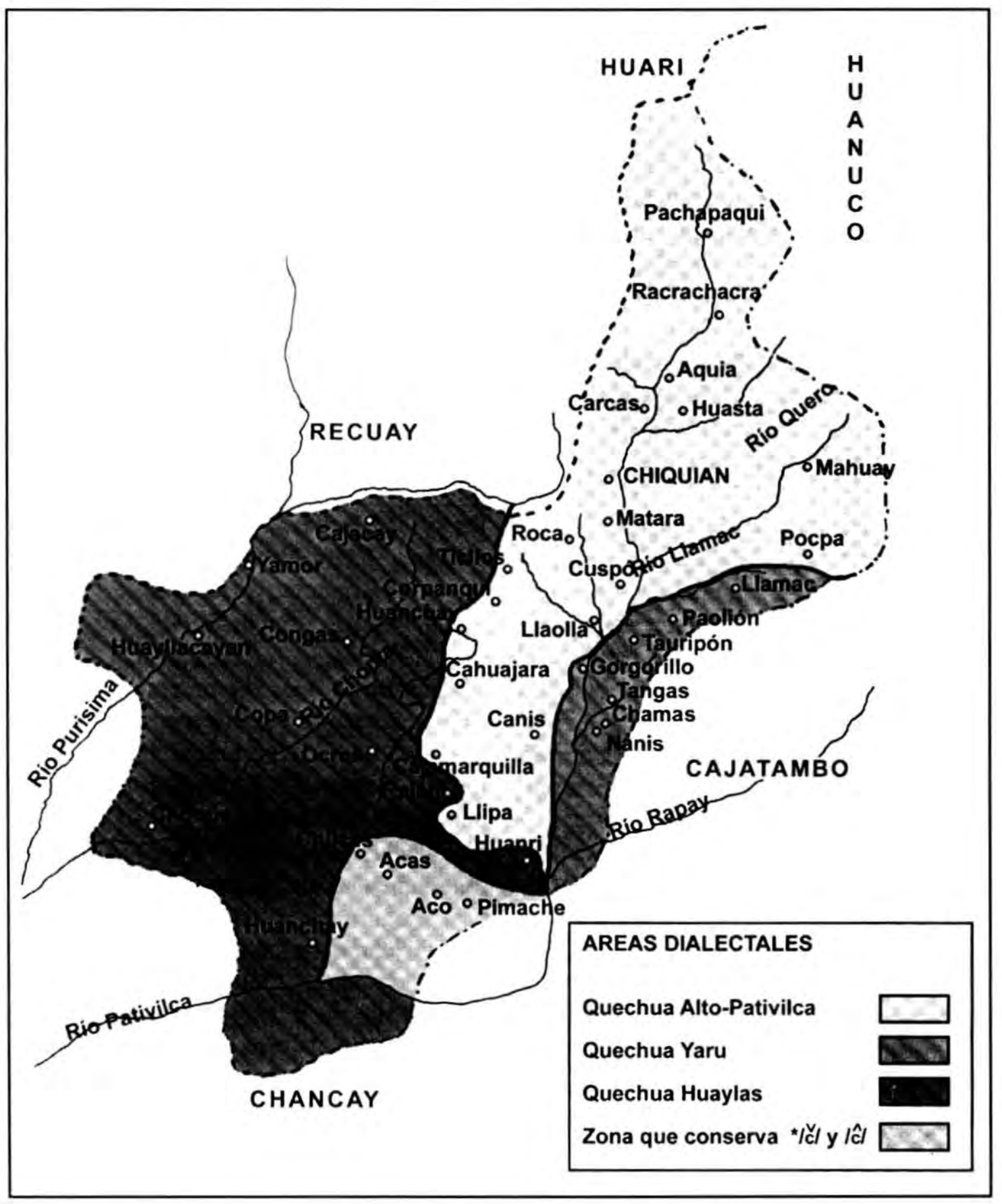

Figura 2. Areas dialectales determinadas. 
se cambia a [æy]; en el habla de los jóvenes, $y / u+y /$ se monoptonguiza opcionalmente. En otras /ay/ - --> [E:] y /u+y/ > [i:].

Dentro de la clasificación de Torero (1974), esta zona dialectal formaría parte del territorio de la variedad Huaylas del dialecto Huaylas-Conchucos.

\section{ZONA DIALECTAL IV}

El quechua de los pueblos de Chilcas, Acas, Aco y Pimache conserva las africadas del proto-quechua: palatal $/ * c$ č/ y palatal retrofleja $/ * \hat{c} /$. Fonéticamente /ĉ/ tiende a ser prepalatal. $\mathrm{El}$ quechua de estos cuatro pueblos usa el pluralizador/-ya:/ y también/-rka:/; como un hecho singular anotamos la coocurrencia en el habla de varios informantes de Chilcas, de los dos pluralizadores, ejemplo:

/carkayka:ya:mun/ "están llegando".

Como morfema de segunda persona usan /-nki/ y /-yki/. En estos lugares se monoptonguiza /aw/ en los sufijos /čaw/ y /-naw/; aparte de esto no se da más este proceso. La consonante /q/ se fricativiza en final de sílaba y el alargamiento es estacionario. Se despalataliza $/ * \tilde{n} /$ pero se conserva $/ *$ ll $/$ en raíces.

De acuerdo con Torero (1974), por conser$\mathrm{var} / * \mathrm{c} / \mathrm{y} / * \hat{\mathrm{c}} /$ en su sistema fonológico, el quechua de estos lugares sería semejante al habla de Sihuas, perteneciente al dialecto Conchucos de la variedad Huaylas - Conchucos.

\section{NOTAS}

'El concepto zonificación ha sido definido por Alberto Escobar (1976:38) en los siguientes términos: "Por zonificación comprendemos el intento de demarcar aproximadamente las áreas espaciales en las que prevalece un determinado tipo o una espe- cífica variedad. Significa lo anterior que en ciertos dominios geográficos reconocemos la prevalencia de algunas formas o haz de rasgos lingüísticos; [...]. En verdad, de lo que se trata es de señalar la trama de notas comunes o zonales, que nos permite definir el perfil predominante en las comunidades enmarcada dentro de un espacio geográfico".

${ }^{2}$ El nombre Quechua I acuñado por A. Torero es equivalente del nombre Quechua B en la terminología de G. Parker (1971).

${ }^{3}$ Los siguientes lugares de la zona sur de Ancash figuran en el mapa: Acas, Aco, Aquia; Cajacay, Cajamarquilla, Canis, Carcas, Carhuajara; Chamas, Chilcas, Chiquián, Choque; Congas, Copa, Corpanqui, Cuspón Gorgorillo, Huanchay (de Ocros), Huanchay, Huanri, Huasta, Huayllacayán; Llaclla, Llamac, Llipa; Mahuay, Mangas, Matara; Nanis; Ocros, Pachapaqui, Pacllón, Pimache, Pocpa; Racrachaca, Raján, Roca; Tauripón, Ticllos; Yamor.

Algunos lugares de la lista precedente son capitales de distrito, otros son simplemente anexos. La ubicación aproximada de cada lugar la tenemos en el mapa, en el que cada uno de los nombres va acompañado con una letra del alfabeto que lo va a representar en los cuadros subsiguientes.

${ }^{4}$ Históricamente, como ya hemos anotado en otra parte, el territorio de la provincia de Bolognesi y Ocros formaba parte, hasta 1903, de la provincia limeña de Cajatambo. Más importante que esto para explicarnos las semejanzas lingüísticas entre las hablas limítrofes de las dos provincias son las múltiples coincidencias etnoculturales que trasuntan las estrechas relaciones socioeconómicas que hasta ahora mantienen Cajatambo y los distritos vecinos de Pacllón y San Francisco de Mangas. Por otro lado, se sabe que desde 
muy tempranos años de la Colonia, el distrito San Francisco de Mangas constituía un centro de adoctrinamiento religioso (Doctrina de Mangas) que comprendía precisamente a los poblados de Pacllón, Tauripón, Gorgorillo, Chamas, Nanis
(Bolognesi) y Copa, Pocquián y Huayllapa (Cajatambo). Hasta ahora permanecen en el pueblo de Mangas las capillas (pequeñas iglesias) y las casas de hospedaje de algunos de los pueblos que formaban parte de la "doctrina".

\section{BIBLIOGRAFía}

ADELAAR, W. F. H.

1970 Vowel quantity in wanka verb suffixes. XXXIX Congreso de Americanistas. IEP Organizador.

\section{CARRILLO RAMÍREZ, Alberto}

1953 Ensayo monográfico de la provincia de Bolognesi (Homenaje al aniversario de su creación). Libro II. Tipografía Córdova. Arequipa.

CERRÓN-PALOMINO, Rodolfo 1973 La evolución del fonema /q/ en Ya>a Wanka. Lima: CILA-UNMSM. Doc. $\mathrm{N}^{\circ} 15$.

DE CHENE, Brent

1979 The historical Phonology of vowel Length. UCLA, Ph.D. Dissertation.

ESCOBAR, Alberto 1978 Variaciones sociolingüísticas del castellano en el Perú. Lima. IEP.
PANTOJA, S.; J. RIPKENS y W. SWISSHELM

1974 Cuentos y relatos en el quechua de Huaraz. I, II. Estudios Culturales Benedictinos, No. 3. Huaraz.

\section{PARKER, Gary}

1971 Comparative Quechua Phonology and Grammar V. The evolution of Quechua B. Working Paper in Linguistics. Hawaii.

SOLÍS F., Gustavo

1976 Zonificación dialectal del quechua de la provincia de Bolognesi (Ancash). Tesis UNMSM. Lima.

TORERO, Alfredo 1964 Los dialectos quechuas. Anales Científicos de la Universidad Agraria. Lima.

1974 El quechua y la historia social andina. Universidad Ricardo Palma. Lima. 


\begin{tabular}{|c|c|c|c|c|c|}
\hline Nanis & - & + & & & t \\
\hline Chamas & I & + & & & + \\
\hline Mangas & 0 & + & & & + \\
\hline Gorgorillo & $\infty$ & $t$ & & & + \\
\hline Tauripón & 匆 & + & & & + \\
\hline Pacllón & A & + & & & + \\
\hline Llamac & 颍 & + & + & + & + \\
\hline Pocpa & $u$ & + & + & + & + \\
\hline Mahuay & $\infty$ & + & + & + & + \\
\hline Huasta & $\varangle$ & + & + & + & + \\
\hline Aquia & $\mathrm{N}$ & + & + & + & + \\
\hline Racrachaca & $x$ & + & + & + & + \\
\hline Pachapaqui & $x$ & + & + & + & + \\
\hline Carcas & $B$ & + & + & + & + \\
\hline Chiquián & $>$ & + & + & + & + \\
\hline Matara & 2 & + & + & + & + \\
\hline Roca & $H$ & + & + & + & + \\
\hline Cuspón & n & + & + & + & + \\
\hline Llaclla & द्य & + & + & + & + \\
\hline Ticllos & 4 & + & + & + & + \\
\hline Corpanqui & $\sigma$ & + & + & + & + \\
\hline Huanchay & a & + & + & + & + \\
\hline Carhuajara & 0 & + & + & + & + \\
\hline Canis & 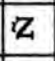 & + & + & + & + \\
\hline Cajamarquilla & $\mathbf{Z}$ & + & + & + & + \\
\hline Llipa & $\bar{z}$ & + & + & + & + \\
\hline Pimache & 격 & + & + & + & + \\
\hline Aco & H & + & + & + & + \\
\hline Acas & $\square$ & + & + & + & + \\
\hline Chilcas & $\neg$ & + & + & + & + \\
\hline Huanri & -1 & + & + & + & - \\
\hline Raján & 4 & + & + & + & $\sim$ \\
\hline Huanchay & 0 & + & + & + & $\sim$ \\
\hline Ocros & 4 & + & + & + & $\sim$ \\
\hline Cajacay & $m$ & + & + & + & n \\
\hline Yamor & A & + & + & + & a \\
\hline Huayllacaján & 起 & + & + & + & $\sim$ \\
\hline Congas & 0 & + & + & + & $\sim$ \\
\hline Copa & $m$ & + & + & + & $\sim$ \\
\hline \multirow[t]{2}{*}{ Choque } & $<$ & + & + & + & م \\
\hline & & 촛 & 矛 & 찻 & 离 \\
\hline
\end{tabular}




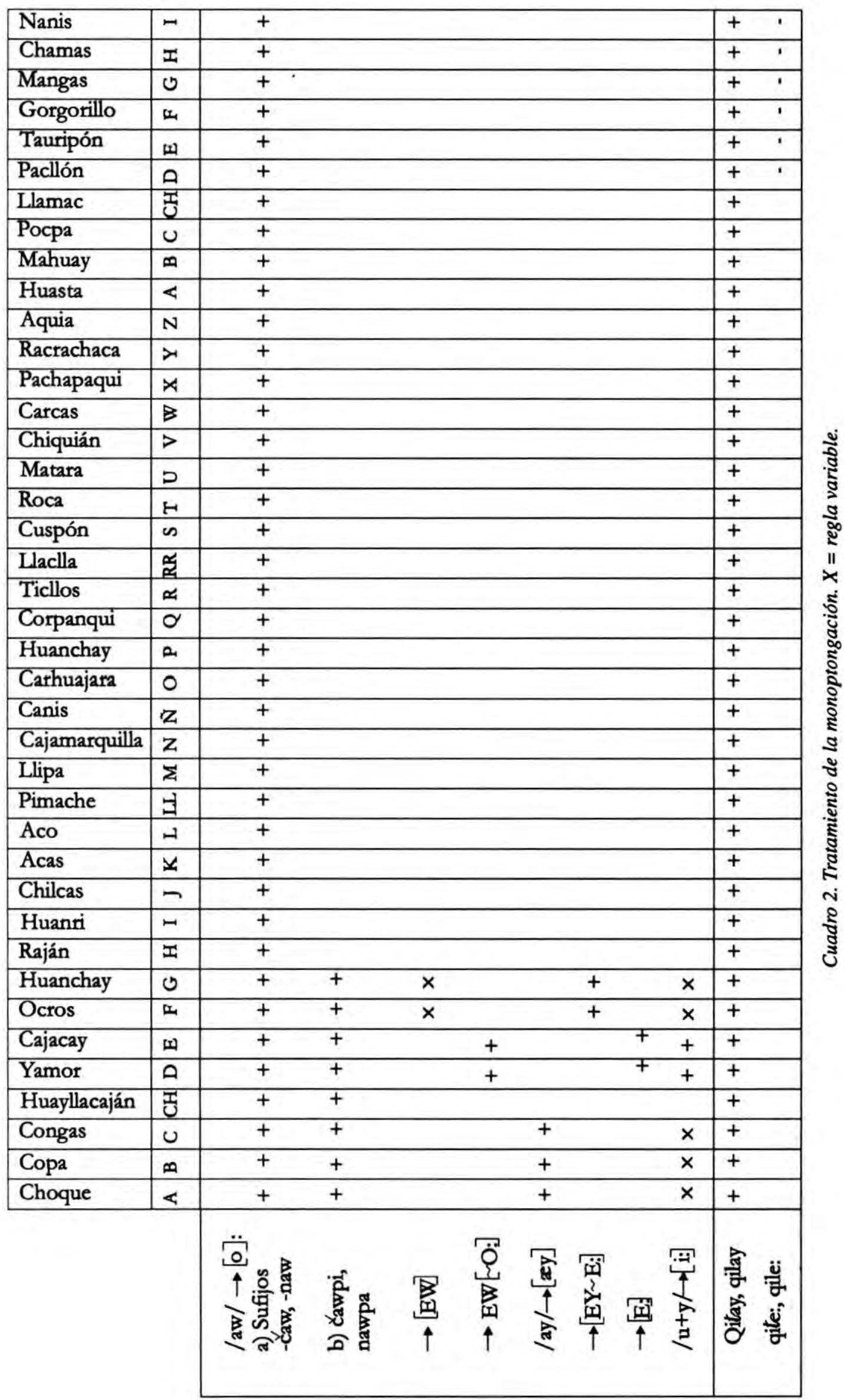




\begin{tabular}{|c|c|c|c|c|c|c|c|c|}
\hline Nanis & $\mapsto$ & + & + & + & + & + & + & + \\
\hline Chamas & I & + & + & + & + & + & + & + \\
\hline Mangas & 0 & + & + & + & + & + & + & + \\
\hline Gorgorillo & $\infty$ & + & + & + & + & + & + & + \\
\hline Tauripón & m & + & + & + & + & + & + & + \\
\hline Pacllón & a & + & + & + & + & + & + & + \\
\hline Llamac & 甹 & + & + & + & + & + & + & + \\
\hline Pocpa & 0 & + & + & + & + & + & + & + \\
\hline Mahuay & $\infty$ & + & + & + & + & + & + & + \\
\hline Huasta & $\ll$ & + & + & + & + & + & + & + \\
\hline Aquia & $N$ & + & + & + & + & + & + & + \\
\hline Racrachaca & 7 & + & + & + & + & + & + & + \\
\hline Pachapaqui & $x$ & + & + & + & + & + & + & + \\
\hline Carcas & $B$ & + & + & + & + & + & + & + \\
\hline Chiquián & $>$ & + & + & + & + & + & + & + \\
\hline Matara & $p$ & + & + & + & + & + & + & + \\
\hline Roca & $H$ & + & + & + & + & + & + & + \\
\hline Cuspón & $\infty$ & + & + & + & + & + & + & + \\
\hline Llaclla & 蛋 & + & + & + & + & + & + & $x$ \\
\hline Ticllos & $\infty$ & + & + & + & + & + & + & $x$ \\
\hline Corpanqui & $\alpha$ & + & + & + & + & + & + & $x$ \\
\hline Huanchay & A & + & + & + & + & + & + & $x$ \\
\hline Carhuajara & 0 & + & + & + & + & & ' & 1 \\
\hline Canis & Z & + & + & + & + & & ' & ' \\
\hline Cajamarquilla & z & + & + & + & + & & ' & ' \\
\hline Llipa & $\sum$ & + & + & + & + & & ' & 1 \\
\hline Pimache & 可 & . & . & & + & & ' & $\therefore$ \\
\hline Aco & $H$ & . &. & & + & & ' & ' \\
\hline Acas & $x$ & . & . & & + & & ' & i \\
\hline Chilcas & $\neg$ & ' & 1 & & + & & ' & ' \\
\hline Huanri & $\mapsto$ & + & + & ' & + & & ' & 1 \\
\hline Raján & 巫 & + & + & ! & + & & ' & 1 \\
\hline Huanchay & 0 & + & + & ' & + & & ' & . \\
\hline Ocros & 山 & + & + & ' & + & & ' & ' \\
\hline Cajacay & (4) & + & + & . & + & & 1 & 1 \\
\hline Yamor & A & + & + & ' & + & & , & ' \\
\hline Huayllacaján & 誌 & + & + & $\cdot$ & + & & ' & ' \\
\hline Congas & $u$ & + & + & . & + & & ' & ' \\
\hline Copa & $\infty$ & + & + & ' & + & & . & · \\
\hline \multirow[t]{2}{*}{ Choque } & $<$ & + & + & ' & + & & . & . \\
\hline & & $\underbrace{\infty}_{* 0}$ & $\frac{i}{* 0}$ & $\begin{array}{l}n \\
1 \\
3\end{array}$ & 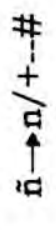 & $\underset{+}{+\#}$ & $\begin{array}{l}\# \\
+ \\
+ \\
+ \\
+\end{array}$ & 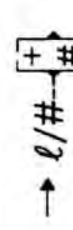 \\
\hline
\end{tabular}

